\title{
Conjugated Linoleic Acid in Rumen Liquor: Their Relation with Other Carbon 18 Fatty Acids and Rumen pH
}

\begin{abstract}
This study was focused on the quantitative analysis of Conjugated Linoleic Acids (CLAs) in rumen liquor and assessment of the relationship between CLAs and other carbon 18 fatty acids and rumen $\mathrm{pH}$ of cattle slaughtered for meat consumption. The fatty acids were extracted using modified Folch method and determined by gas chromatography. The mean (n = 22) level of CLAs in the rumen liquor was $14.97 \pm 7.46 \mathrm{mg} / 100 \mathrm{~mL}$. Cis-9, trans-11(c9, t11) was the dominant isomer of CLA followed by trans-10,cis-12 (t10, c12). The level of CLAs was positively correlated with the concentration of octadecatrienoic (18:3), cis-9, cis12 octadecadienoic (18:2), trans-11octadecenoic (18:1) and octadecenoic (18:0) acids. Ruminal pH was positively correlated with the amount of c9, t11 and total CLA and negatively correlated with t10, c12 CLA isomer. All these correlations and relationships indicated that CLAs production in the rumen is dependent on availability of carbon 18 fatty acids and $\mathrm{pH}$ of the rumen liquor.
\end{abstract}

Keyword: Conjugated Linoleic Acids, Kedah-Kelantan cattle, gas chromatography 